\title{
The JAK2/STAT3 pathway inhibitor, AG490, suppresses the abnormal behavior of keloid fibroblasts in vitro
}

\author{
YING ZHOU ${ }^{1,2^{*}}$, YUEXIN SUN $^{3 *}$, WENJUN HOU ${ }^{3}$, LIWEN MA $^{3}$, YUE TAO $^{3}$, \\ DAN $\mathrm{LI}^{3}, \mathrm{CUI}^{\mathrm{X}} \mathrm{U}^{3}, \mathrm{JUN} \mathrm{BAO}^{3}$ and WEIXIN FAN ${ }^{2}$ \\ ${ }^{1}$ Department of Dermatology, Drum Tower Clinical Medical College, Nanjing Medical University, Nanjing, Jiangsu 210008; \\ ${ }^{2}$ Department of Dermatology, Jiangsu Province People's Hospital and Nanjing Medical University First Affiliated Hospital, \\ Nanjing, Jiangsu 210029; ${ }^{3}$ Department of Dermatology, Nanjing University Medical School \\ Affiliated Nanjing Drum Tower Hospital, Nanjing, Jiangsu 210008, P.R. China
}

Received October 17, 2019; Accepted April 9, 2020

DOI: $10.3892 /$ ijmm.2020.4592

\begin{abstract}
AG490 is a selective inhibitor of the Janus kinase 2 (JAK2)/signal transducer and activator of transcription 3 (STAT3) signaling pathway. The present study examined its effects on the abnormal behavior of human keloid fibroblasts (HKFs) and evaluated its potential use in the treatment of keloids. Human normal fibroblasts (HNFs) and HKFs were treated with increasing concentrations of AG490. The proliferation of HNFs and HKFs was inhibited by AG490 in both a time- and concentration-dependent manner by increasing apoptosis and inducing G1 cell cycle arrest. The downregulation of cyclin D1 and connective tissue growth factor (CTGF) expression was associated with a decrease in STAT3 expression in response to AG490. The effects of AG490 on TGF- $\beta$-stimulated fibroblasts, including HNFs, HKFs and hypertrophic scar fibroblasts (HSFs) were also evaluated. The TGF- $\beta 1$-stimulated excessive proliferation and CTGF production were markedly inhibited by the application of AG490 in the HNFs, HSFs and HKFs. In addition, the STAT3-specific decoy oligodeoxynucleotides (SODNs) were transfected into HKFs. The invasive ability of the SODN-transfected HKFs was determined and the expression of extracellular matrix
\end{abstract}

Correspondence to: Professor Jun Bao, Department of Dermatology, Nanjing University Medical School Affiliated Nanjing Drum Tower Hospital, 321 Zhongshan Road, Nanjing, Jiangsu 210008, P.R. China

E-mail: 25993598@qq.com

Professor Weixin Fan, Department of Dermatology, Jiangsu Province People's Hospital and Nanjing Medical University First Affiliated Hospital, 300 Guangzhou Road, Nanjing, Jiangsu 210029, P.R. China E-mail: 671610485@qq.com

*Contributed equally

Key words: keloid fibroblast, AG490, signal transducer and activator of transcription 3 , connective tissue growth factor, cell proliferation, apoptosis, cell invasion components was quantified. Similarly, SODNs blocked the constitutive activation of STAT3. SODNs inhibited the invasion and progression of HKFs, possibly via the upregulation of the expression of tissue inhibitor of metalloproteinase-2 (TIMP-2), and the downregulation of the expression of matrix metalloproteinase-2 (MMP-2) and vascular endothelial growth factor (VEGF). On the whole, the findings of the present study demonstrate that STAT3-specific elimination, such as the application of AG490 and decoy ODNs, may serve as promising therapeutic strategies for the treatment of keloids.

\section{Introduction}

Keloid disease (KD) is a complex fibroproliferative disorder which appears as a pathological response to cutaneous wound healing (1). Keloids are defined as persistent existing scars that spread beyond the boundaries of the original wound (2). Keloid scars not only affect the appearance of the skin, but also cause itching and pain, and may lead to necrosis, hemorrhaging and suppuration (1). Histologically, keloids are characterized by the excessive accumulation of extracellular matrix (ECM) components, such as type I collagen (3). Asians are susceptible to keloids, with an incidence rate of $4-16 \%$ (4). KD is difficult to treat, since keloids are prone to recurrence following surgical resection and continue to proliferate for decades. Multiple therapies have been established for KD; however, none of these are fully effective $(2,3,5,6)$. Keloids are considered to be benign tumors of the dermis (7). The development of novel treatment strategies for KD is thus required.

Although the etiopathogenesis of KD remains incompletely understood, keloid fibroblasts (KFs) are deemed as the main inductive cells for keloid formation. KFs exhibit a marked infiltration in lesion tissue and display an increased rate of proliferation; the aberrant behavior of KFs is considered to contribute to disease progression (8). KFs are implicated as mediators of elevated growth factor, chemokine or cytokine production, as well as of the excessive accumulation of ECM components, such as type I/III collagen and fibronectin $(9,10)$. The suppression of the malignant proliferation of KFs and the blocking of their abnormal secretion of growth factors and ECM components is crucial for KD treatment. 
The Janus kinase (JAK)/signal transducer and activator of transcription (STAT) signaling pathway is an important pathway that is responsible for cell signal transduction from the surface into the nucleus. STAT3 is a key member belonging to the JAK/STAT signaling pathway, which plays a leading role in the modulation of diverse processes, including cell proliferation, migration, cytokine or chemokine production and inflammation (11). It has been reported that the expression and phosphorylation of STAT3 is abnormally increased in keloid tissues, suggesting a role of STAT3 in the pathogenesis of keloids (12). STAT3 is in turn subject to multiple regulations by various cytokines, such as interleukin (IL)-6, IL-10 and interferon (IFN)- $\alpha / \gamma$ (13), which were largely produced during the progression of KD. The RNA interference of STAT3 has also been reported to be associated with the inhibition of the proliferation of keloids (12). As STAT3 is not essential for the functioning of normal cells (14), it is rational to consider it as a valuable target for the treatment of diseases, such as KD. However, due to the lack of specific inhibitors, strategies have to be developed to target the STAT3signaling pathway. AG490, as a tyrosine kinase inhibitor for JAK2, can decrease the phosphorylation of STAT3 by selectively inactivating JAK2 (15). The therapeutic potential of AG490 has been illustrated in brain hemorrhage (16), liver injury and fibrosis (17). It thus worthwhile to determine whether it has a special role in the treatment of KD.

The present study hereby investigated the mechanisms through which the inhibition of the JAK2/STAT3 pathway contributes to the regulation of the aberrant behavior of KFs in vitro. The effects of AG490 on the cellular and molecular behavior of human KFs (HKFs), human normal fibroblasts (HNFs) and hypertrophic scar fibroblasts (HSFs) were further examined.

\section{Materials and methods}

Reagents and antibodies. AG490 was purchased from MedChem Express Inc. Stocking solution of AG490 was prepared as $100 \mathrm{mmol} / \mathrm{l}$ using dimethylsulfoxide (DMSO) (Sigma-Aldrich; Merck KGaA) and stored at $-20^{\circ} \mathrm{C}$. AG490 stock solution was further diluted in cell culture medium to various concentrations $(0,12.5,25,50,75$ and $100 \mu \mathrm{mol} / \mathrm{l})$ according to previous reports $(15,18-21)$. The final concentration of DMSO was not $>0.1 \%$. The recombinant human TGF- $\beta$ was purchased from PeproTech Inc. TGF- $\beta$ powder was dissolved by $10 \mathrm{mmol} / \mathrm{l}$ citric acid to prepare a liquid at $1.0 \mathrm{mg} / \mathrm{ml}$ (pH 3.0, containing 0.1\% BSA). Long-term storage was achieved by refrigeration $-20^{\circ} \mathrm{C}$. According to the experiment requirements, TGF- $\beta$ was diluted in cell culture medium to a final concentration of $5 \mu \mathrm{g} / \mathrm{ml}$. TGF- $\beta$ was used to treat the cells $2 \mathrm{~h}$ prior to the application of AG490.

Primary antibodies were purchased from Abcam, including antibodies against STAT3 (ab119352), phosphorylated (p-)STAT3 (Tyr705) (ab76315), CTGF (ab6992) and $\beta$-actin (ab179467). Horseradish peroxidase (HRP)-conjugated secondary antibodies produced in rabbit (SAB1301585) and mouse (SAB1411905) were purchased from Sigma-Aldrich; Merck KGaA. The primers used in this study were synthesized by Takara Biotechnology Co., Ltd (Dalian, China). Other chemical reagents without special indication were obtained from Sigma.

Cell culture. The cell lines, including HNFs, HKFs and HSFs were kind gifts from Dr Qian Tan (Department of Burns and Plastic Surgery, Nanjing University Medical School Affiliated Nanjing Drum Tower Hospital) $(22,23)$. Cells were routinely cultivated in DMEM (HyClone; GE Healthcare Life Sciences) supplemented with $10 \%$ fetal bovine serum (FBS; Invitrogen; Thermo Fisher Scientific, Inc.), $100 \mu \mathrm{g} / \mathrm{ml}$ streptomycin and $100 \mathrm{U} / \mathrm{ml}$ penicillin (Invitrogen; Thermo Fisher Scientific, Inc.) $\left(37^{\circ} \mathrm{C}, 5 \% \mathrm{CO}_{2}\right)$.

Cell proliferation assay (CCK-8 assay). Cells were plated overnight in a 96 -well plate $\left(5 \times 10^{4} /\right.$ well, $\left.100 \mu \mathrm{l}\right)$ until $80 \%$ confluence was achieved. The cells were then treated with $0.1 \%$ DMSO (vehicle control) or AG490 at various concentrations $(0,12.5,25,50,75$ and $100 \mu \mathrm{mol} / \mathrm{l})$ for 24,48 or $72 \mathrm{~h}$. The culture supernatant was discarded and the cells were washed with DMEM once. Following $2 \mathrm{~h}$ of incubation at $37^{\circ} \mathrm{C}$ with $10 \mu 1$ CCK-8 (Sigma-Aldrich; Merck KGaA), the OD absorbance was recorded at $450 \mathrm{~nm}$ using a microplate reader (Tecan Group Ltd.). Cell viability was measured using the following formula: Cell viability $\%=\left[\left(\mathrm{OD}_{\text {test }}-\mathrm{OD}_{\text {blank }}\right) /\left(\mathrm{OD}_{\text {control }}-\mathrm{OD}_{\text {blank }}\right)\right]$ $\mathrm{x} 100 \%$.

Cell cycle assay. During the exponential growth phase, HKFs were digested by $0.25 \%$ trypsin and suspended in DMEM containing no FBS. Cells were plated in a 6-well plate $\left(2 \times 10^{6} /\right.$ well, $\left.2 \mathrm{ml}\right)$ followed by $24 \mathrm{~h}$ of serum starvation. Subsequently, the cells were treated with the vehicle control (0.1\% DMSO) or various concentrations $(12.5,25,50,75$ and $100 \mu \mathrm{mol} / \mathrm{l}$ ) of AG490 for $24 \mathrm{~h}$. Following treatment, cells were harvested and washed with cold PBS, followed by fixation in $70 \%$ ethanol at $4^{\circ} \mathrm{C}$ overnight. Fixed cells were then treated with RNase $(100 \mu \mathrm{g} / \mathrm{ml}, 50 \mu \mathrm{l})$ at $37^{\circ} \mathrm{C}$ in the dark for $30 \mathrm{~min}$ and stained with propidium iodide (PI, $200 \mu \mathrm{l}$ from $50 \mu \mathrm{g} / \mathrm{ml}$ stock solution) at room temperature for $5 \mathrm{~min}$. Cell cycle assay was performed using a flow cytometer (BD Biosciences). Data were analyzed using FlowJo7.6 software (Tree Star, Inc.).

Annexin-V/PI binding apoptosis assay. The serum-starved cells were prepared and cultured as described above. Following treatment with AG490 at the indicated concentrations for $24 \mathrm{~h}$, cells were washed with cold PBS 3 times, and then incubated with Annexin V-FITC and PI at room temperature in the dark for $15 \mathrm{~min}$. Samples were analyzed by flow cytometry (BD Biosciences) within $1 \mathrm{~h}$. Data were analyzed using FlowJo7.6 software (Tree Star, Inc.).

Western blot analysis. Following proper treatment, cells were collected and lysed in RIPA buffer (WEIAO BioTech Co. Ltd.) with $1 \mathrm{mmol} / \mathrm{l}$ PMSF. The same amount $(30 \mu \mathrm{g})$ of proteins was loaded on 10\% SDS/PAGE gels and transferred to PVDF membranes $(0.45 \mu \mathrm{M})$. After blocking with 5\% skim milk in the TBST bufer $(50 \mathrm{mmol} / \mathrm{l}$ Tris- $\mathrm{HCl}, 150 \mathrm{mmol} / \mathrm{l}$ $\mathrm{NaCl}, 0.1 \%$ Tween-20, $\mathrm{pH}$ 7.4) at room temperature for $1 \mathrm{~h}$, the membranes were incubated with primary antibodies $(1: 1,000)$ overnight at $4^{\circ} \mathrm{C}$. The membranes were then washed with TBST buffer and incubated with horseradish peroxidase 
(HRP)-conjugated secondary antibodies (1:20,000) for $1 \mathrm{~h}$ at room temperature. The chemiluminescence detection kit (Thermo Fisher Scientific, Inc.) was used to detect the protein signals with an X-ray film (Fuji Film). The quantification of the western blots was performed using ImageJ $1.52 \mathrm{u}$ software. The expression levels of each protein were normalized to those of corresponding $\beta$-actin, respectively.

RNA isolation, reverse transcription and quantitative PCR. Differently treated cells were washed with PBS and collected respectively. Total RNA was isolated using TRIzol Reagent (Invitrogen; Thermo Fisher Scientific, Inc.). cDNA was prepared using the ReverTra Ace qPCR RT kit (Toyobo) and amplified by real-time PCR on StepOne Plus (Thermo Fisher Scientific, Inc.). The primers sets and sequences are presented in Table I. Reactions were run in duplicate as follows: $95^{\circ} \mathrm{C}$ for $5 \mathrm{~min}$, followed by 40 cycles of $15 \mathrm{sec}$ at $95^{\circ} \mathrm{C}$ and $1 \mathrm{~min}$ at $60^{\circ} \mathrm{C}$. The ending cycle was maintained at $60^{\circ} \mathrm{C}$ for $5 \mathrm{~min}$. Gene expression levels were determined by relative quantification according to the $\Delta \Delta \mathrm{Cq}$ method as previously described (24).

STAT3 decoy and scramble oligodeoxynucleotides (ODNs). STAT3 decoy and scrambled control ODNs were designed as previously described (25) and were synthesized by Sangon Biotechnology Co., Ltd. The STAT3 decoy ODN sequence (SODNs) was 5'-CATTTCCCGTAAATC-3' and 3'-GTAAAGGGCATTTAG-5'. The scrambled ODN sequence (MODN) was 5'-CATTTCCCTTAAATC-3' and 3'-GTAAAG GGAATTTAG-5'. The ODNs and the Lipofectamine ${ }^{\mathrm{TM}} 2000$ (Invitrogen; Thermo Fisher Scientific, Inc.) were respectively diluted in equal volumes of serum-free medium before being mixed at a ratio of 2:5 ( $\mu \mathrm{g}: \mu \mathrm{l})$. The mixture was incubated for $20 \mathrm{~min}$ at room temperature and then transfected into the cells.

Cell invasion assay. Cell invasion assay in vitro was performed using a 24-well Transwell chamber (Corning, Inc.) with an $8-\mu \mathrm{m}$ pore size polycarbonate filter coated with ECMatrix gel (Chemicon; Thermo Fisher Scientific, Inc.) to form a continuous thin layer. HKFs transfected with ODNs (1.0x10\%sample) were harvested in serum-free DMEM containing 0.1\% BSA and added to the upper chamber. The lower chamber contained $500 \mu \mathrm{l}$ DMEM and 5\% FBS. Cells were incubated for $72 \mathrm{~h}$ $\left(37^{\circ} \mathrm{C}, 5 \% \mathrm{CO}_{2}\right)$ followed by complete removal from the upper surface of the filter using cotton swabs. The filters were fixed in $95 \%$ ethanol and stained with crystal violet (Sigma-Aldrich; Merck KGaA) at room temperature for $20 \mathrm{~min}$. Cells migrating across the Matrigel and reaching the lower surface of the filter were counted under a light microscope [Nikon Imaging (China) Sales Co., Ltd.]. Samples were acquired in triplicate and data were measured as the average cell number in 10 fields.

Electrophoretic mobility shift assay (EMSA). After the cells were transfected with ODNs for $72 \mathrm{~h}$ as described above, the nuclear protein was prepared as reported previously (7). EMSA was performed using the double-stranded synthetic oligonucleotides mimicking the STAT3 binding sites present within the promoters of the c-fos gene as follows: Sense, 5'-AGC TTCATTTCCCGTAAATCCCTA-3' and antisense, 5'-TAG GGATTTACGGGAAATGAAGCT-3'. The synthetic probes were 5'-end labeled using $\gamma-{ }^{32} \mathrm{P}-\mathrm{dATP}$ and T4 polynucleotide
Table I. Primer sequences used in RT-qPCR.

\begin{tabular}{ll}
\hline Primer & \multicolumn{1}{c}{ Sequence $(5 ' \rightarrow 3 ')$} \\
\hline STAT3 forward & CACATGCCACTTTGGTGTTTCA \\
STAT3 reverse & GGGCAATCTCCATTGGCTTC \\
Cyclin D1 forward & TCGGTGTCCTACTTCAAATGTGT \\
Cyclin D1 reverse & GAAGCGGTCCAGGTAGTTCA \\
CTGF forward & CTTGCGAAGCTGACCTGGAA \\
CTGF reverse & AAAGCTCAAACTTGATAGGCTTGGA \\
MMP-2 forward & ACCTACACCAAGAACTTCCGTCTG \\
MMP-2 reverse & CTCGTATACCGCATCAATCT \\
TIMP-2 forward & TCTGGCATCAGGCACCTGGATTGAG \\
TIMP-2 reverse & CTATCCTAACCCCCATATCACT \\
VEGF forward & GCTCTACTTCCCCAAATCACT \\
VEGF reverse & CCCAAAAGCAGGTCACTCACT \\
$\beta$-actin forward & TGGCACCCAGCACAATGAA \\
$\beta$-actin reverse & CTAAGTCATAGTCCGCCTAGAAGCA
\end{tabular}

STAT3, signal transducer and activator of transcription 3; CTGF, connective tissue growth factor; MMP-2, matrix metalloproteinase 2; VEGF, vascular endothelial growth factor; TIMP-2, tissue inhibitor of metalloproteinase-2.

kinase. Nuclear proteins $(10 \mu \mathrm{g})$ from each sample were incubated with $\gamma^{32} \mathrm{P}$-labeled oligonucleotide probe $(0.1 \mu \mathrm{g} / \mu \mathrm{l}$, $1 \mu \mathrm{l}$ ) in $20 \mu \mathrm{l}$ of binding buffer containing $10 \mathrm{mM}$ HEPES (pH 7.8), $50 \mathrm{mmol} / \mathrm{K} \mathrm{KCl}, 1 \mathrm{mmol} / 1$ EDTA, $5 \mathrm{mmol} / 1 \mathrm{MgCl}_{2}$, $10 \%$ glycerol, $5 \mathrm{mmol} / \mathrm{l} \mathrm{DTT}, 1 \mathrm{mg} / \mathrm{ml}$ bovine serum albumin, and $1 \mathrm{mmol} / 1 \mathrm{Na}_{3} \mathrm{VO}_{4}$. Following a 15 -min incubation at room temperature, the samples were separated on a $6 \%$ non-denaturing polyacrylamide gel. For competition analyses, nuclear protein was incubated with cold probe (unlabeled oligonucleotide) for $15 \mathrm{~min}$ at room temperature prior to the addition of the labeled oligonucleotides. Gels were dried and subjected to standard autoradiographic procedures at $-70^{\circ} \mathrm{C}$. The quantification of STAT3 activation levels was performed using Image $1.52 \mathrm{u}$ software.

Statistical analysis. Data are presented as the means \pm SD obtained from at least 3 independent tests. Data were analyzed by one-way ANOVA followed by Tukey's multiple comparisons test (GraphPad Prism 7, GraphPad Prism, Inc.). $\mathrm{P}<0.05$ was considered to indicate a statistically significant difference. The association between mRNA expression levels was examined by a simple linear regression model. Linear regression analysis was performed using SPSS ${ }^{\circledR}$ Statistical software (IBM, Inc.).

\section{Results}

AG490 inhibits the proliferation and induces the G1 cell cycle arrest of HKFs. The effects of AG490 (chemical structure presented in Fig. 1A), the JAK2/STAT3 pathway inhibitor, were examined in HNFs and HKFs. Increasing concentrations $(0,12.5,25,50,75$ and $100 \mu \mathrm{mol} / \mathrm{l})$ of AG490 were respectively prepared to ensure that the final concentration of DMSO in working assays was not $>0.1 \%$. Pre-experimental tests were performed to confirm that DMSO had no deleterious effects on 
A

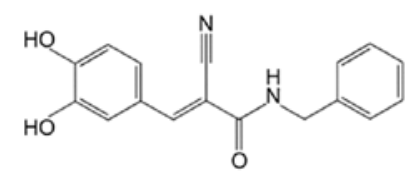

B HNFs

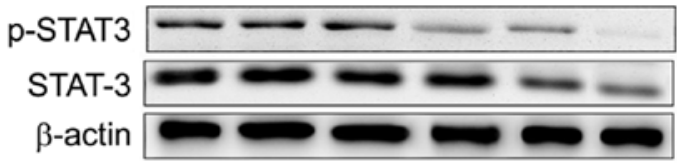

HKFs

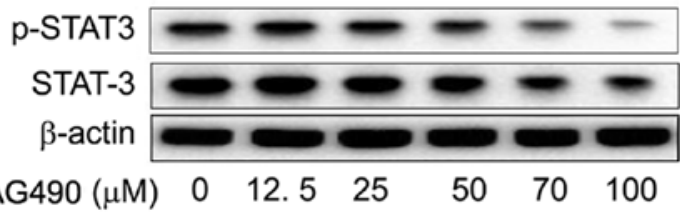

C

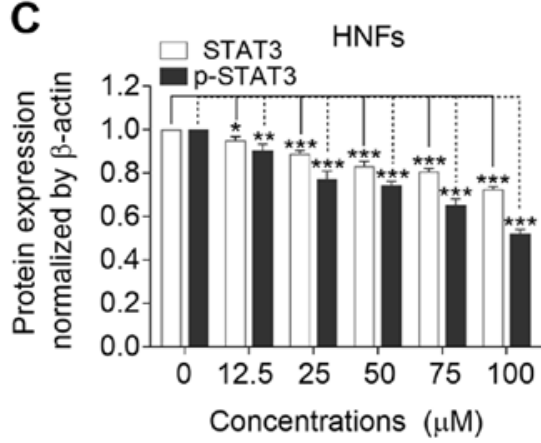

$E$

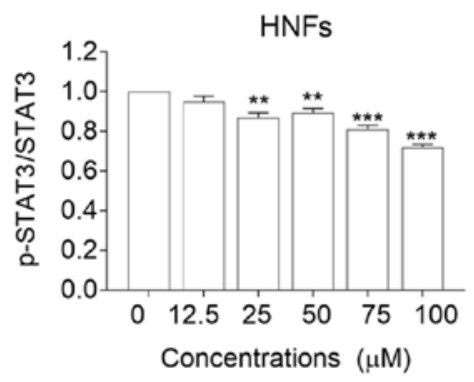

D

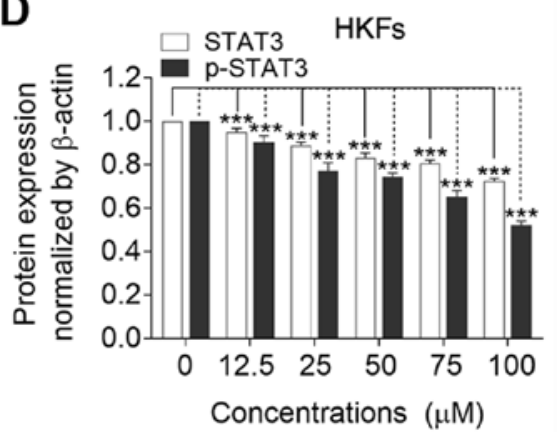

$\mathbf{F}$

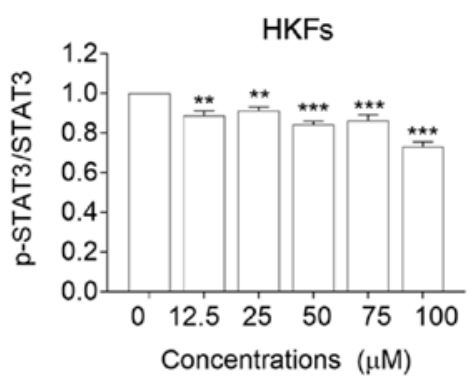

Figure 1. AG490 decreases the expression of both STAT3 and activated STAT3. HNFs and HKFs were treated with $0,12.5,25,50,75,100 \mu \mathrm{mol} / 1$ AG490 for $24 \mathrm{~h}$, respectively. (A) Chemical structure of AG490 is shown. (B) Relative expression of STAT3 and phosphorylated STAT3 (p-STAT3) proteins in HNFs and HKFs detected by western blot analysis. $\beta$-actin was used as a loading control. (C and D) The ratio of total STAT3 and p-STAT3 to $\beta$-actin in HNFs (C) and HKFs (D) was examined by densitometry, respectively. (E and F) The ratio of p-STAT3 to total STATs in HNFs (E) and HKFs (F) was calculated and shown, respectively. Statistical analysis was performed using one-way ANOVA followed by post-hoc Tukey's multiple comparisons test. ${ }^{*} \mathrm{P}<0.05$, ${ }^{* *} \mathrm{P}<0.01$ and ${ }_{* * * *} \mathrm{P}<0.005$ vs. the control; $\mathrm{n}=3$. STAT3, signal transducer and activator of transcription 3; HNFs, human normal fibroblasts; HKFs, human keloid fibroblasts.

cell proliferation at concentrations $<0.2 \%$ (Fig. S1A). As was expected, the expression levels of both STAT3 and p-STAT3 were decreased by the application of AG490 in a dose-dependent manner (Fig. 1). In addition, the HKFs expressed significantly higher mRNA levels of STAT3 than the HNFs, as detected by RT-qPCR (Fig. S1B), which is in accordance with the findings of previous findings (12). Compared with the control group, AG490 inhibited the proliferation of HNFs and HKFs at 24, 48 and $72 \mathrm{~h}$ in a time- and concentration-dependent manner $(\mathrm{P}<0.05$, Fig. 2A). At all testing time points, the inhibition was more evident in the HKFs than the HNFs $(\mathrm{P}<0.05)$. To elucidate the mechanisms through which AG490 suppressed HKF proliferation, the cell cycle distribution was subsequently detected by flow cytometry. Following $24 \mathrm{~h}$ of pre-treatment with AG490 at concentrations up to $100 \mu \mathrm{mol} / 1$, the cell proportion markedly declined from approximately 50 to $25 \%$ at the $\mathrm{G} 2 / \mathrm{M}$ phase, while increased it from 20 to $42 \%$ at the G0/G1 phase in response to AG490 (Fig. 2B and C). By contrast, the cell numbers in the $\mathrm{S}$ phase remained unaltered upon the application of AG490. The results indicate that AG490 mainly induced cell cycle arrest at G1 phase, which is different from most clinical DNA damage drugs that induced $\mathrm{G} 2 / \mathrm{M}$ arrest. A diagram of the cell cycle for each phase based on flow cytometry (Fig. 2C) also revealed that the effective concentration of AG490 to reduce HKF proliferation was at least $50 \mu \mathrm{mol} / 1$.

AG490 induces the apoptosis of HKFs. To demonstrate whether the cytotoxicity of AG490 to HKFs was associated with the induction of apoptosis, HKFs were stained with Annexin V-FITC/PI following exposure to AG490 for $24 \mathrm{~h}$. Cells were harvested and analyzed by flow cytometry. The results were plotted in two-dimensional dot plots, which could be divided in 4 regions (Q1, Q2, Q3 and Q4; Fig. 3). According to a previous report, Annexin V-FITC-positive and PI-negative cells (Annexin V-FITC ${ }^{+} / \mathrm{PI}^{-}$, Q3) are generally considered apoptotic cells in the early stage, while 
A

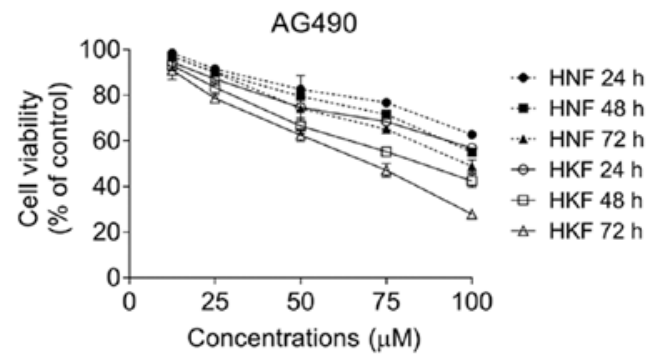

B
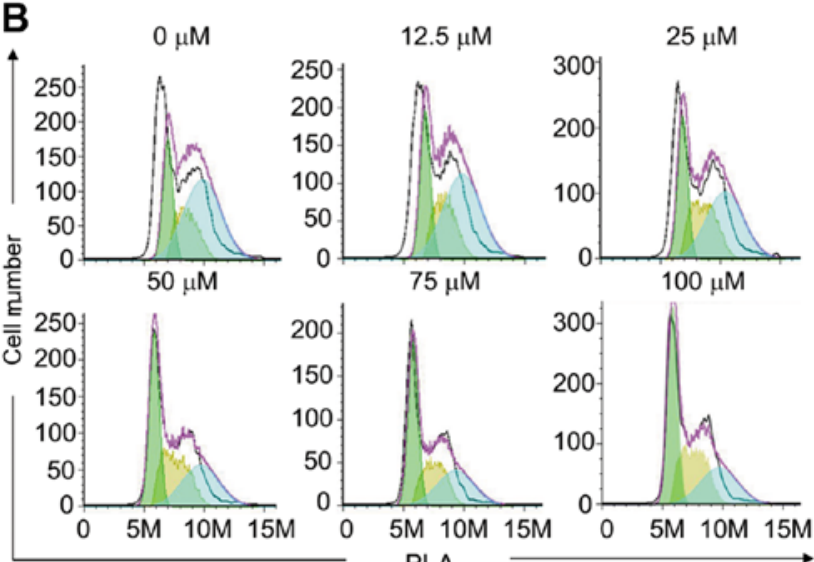

C

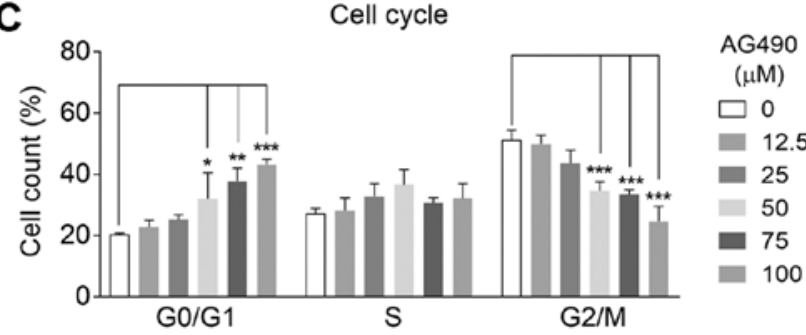

Figure 2. AG490 inhibits the proliferation of fibroblasts and induces G1 phase arrest of HKFs. (A) Proliferation rates of HNFs and HKFs was detected by CCK-8 assay. HNFs and HKFs were treated with $0,12.5,25$, 50, 75, $100 \mu \mathrm{mol} / 1 \mathrm{AG} 490$ for 24, 48 and $72 \mathrm{~h}$, respectively. Compared with the control group, AG490 inhibited the proliferation of HNFs and HKFs in both time- and concentration-dependent manner. (B) The cell numbers in the G0/G1, S, and G2/M phases were analyzed by flow cytometry following treatment with AG490 for $24 \mathrm{~h}$. (C) The total number in different cell cycle phase was measured, respectively. Values represent the means $\pm S D, n=3 /$ each group. ${ }^{*} \mathrm{P}<0.05,{ }^{* *} \mathrm{P}<0.01$ and ${ }^{* * * *} \mathrm{P}<0.005$ vs. the control. HNFs, human normal fibroblasts; HKFs, human keloid fibroblasts.

Annexin V/PI-double-positive cells (Annexin V-FITC ${ }^{+} / \mathrm{PI}^{+}$, Q2) are considered apoptotic cells in the late stage (26). Q1 (Annexin V-FITC//PI') and Q4 (Annexin V-FITC $/ \mathrm{PI}^{-}$) indicate necrotic cells and viable cells, respectively. Consistent with the findings of cell proliferation assay, a significant enhancement of HKF apoptosis was observed when AG490 was used at concentrations of 50,75 and $100 \mu \mathrm{mol} / 1$ (Fig. 3A and B). Specifically, the proportions of HKFs at the early stages of apoptosis were markedly promoted in response to AG490, while those in the late stages of apoptosis were moderately enhanced (Fig. 3C).

AG490 decreases the mRNA levels of CTGF and cyclin D1 in $H K F$. To gain further insight into the mechanisms through which AG490 suppresses the proliferation and induces the G1 phase arrest of HKFs, the expression levels of cyclin D1,
A
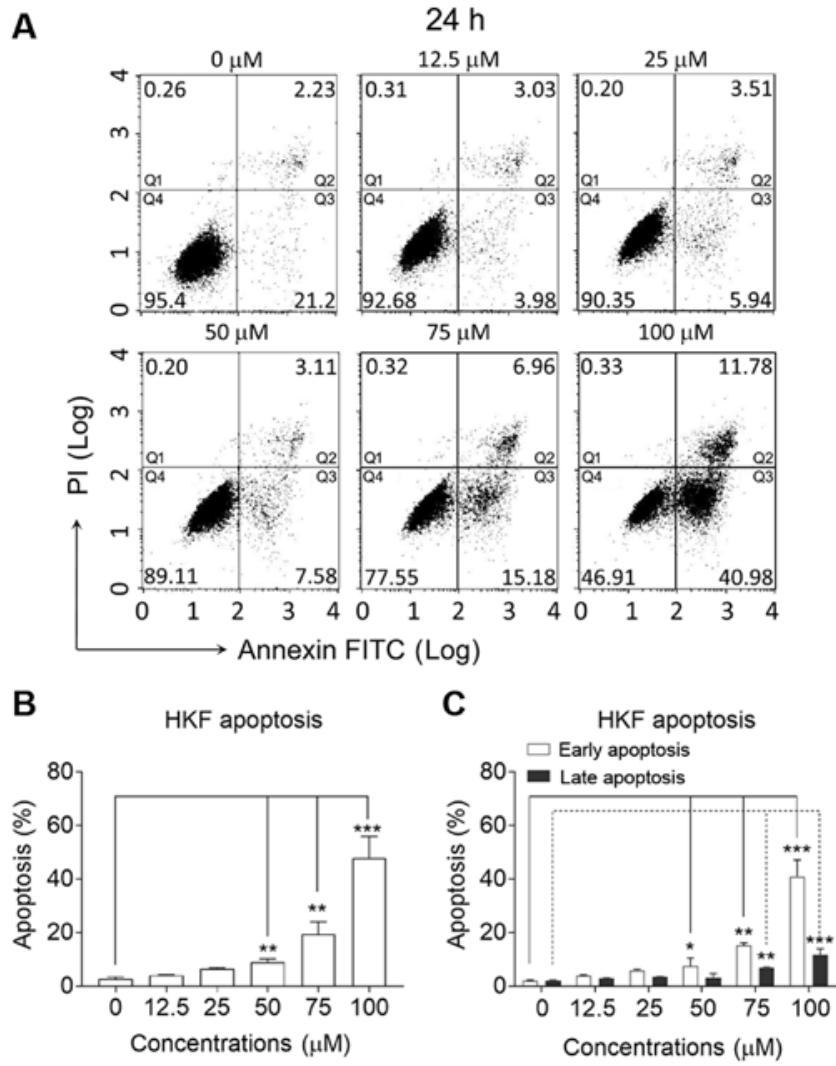

Figure 3. AG490 markedly enhances the apoptosis of HKFs. HKFs were treated with $0,12.5,25,50,75,100 \mu \mathrm{mol} / 1 \mathrm{AG} 490$ for $24 \mathrm{~h}$, respectively. (A) Cell apoptosis was detected by Annexin V/PI double staining assay. The data were generated by flow cytometry are plotted in two-dimensional dot plots, which can be divided into 4 regions (Q1, Q2, Q3 and Q4). (B) The proportion of total apoptotic (Annexin V-positive) cells increased concentration-dependently with AG490 treatment. (C) The proportion of apoptotic cells at the early phase (Q3, Annexin V-positive and PI-negative) and late phase (Q2, Annexin V and PI double-positive). Values represent the means $\pm \mathrm{SD}, \mathrm{n}=3 ;{ }^{*} \mathrm{P}<0.05,{ }^{* *} \mathrm{P}<0.01$ and ${ }^{* * * *} \mathrm{P}<0.005$ vs. control. HKFs, human keloid fibroblasts.

the G1/S cell cycle checkpoint controller and CTGF, the primary regulator of fibroblast proliferation and differentiation, were assessed in HKFs following $24 \mathrm{~h}$ of treatment (Fig. 4). Compared with the control group, the relative mRNA expression levels of STAT3, cyclin D1 and CTGF in the HKFs decreased significantly in response to increasing concentrations of AG490 ( $\mathrm{P}<0.05$, Fig. 4A-C). Moreover, there was a strong association between the mRNA expression levels of STAT3 and cyclin D1 ( $\mathrm{F}=43.647, \mathrm{P}<0.005$; Fig. 4D and E) and CTGF ( $\mathrm{F}=42.138, \mathrm{P}<0.005$; Fig. $4 \mathrm{~F}$ and $\mathrm{G}$ ) as shown by a linear regression analysis. These results indicated that the downregulation of cyclin D1 and CTGF were associated with the AG490-induced decrease in STAT3 expression.

Suppressive effects of AG490 on the excessive proliferation of fibroblasts induced by TGF- $\beta$. It has been well established that TGF- $\beta$ plays a critical role in the pathogenesis of keloids by promoting the proliferation, collagen formation and differentiation of dermal fibroblasts (27). In the present study, to evaluate the effects of AG490 on TGF- $\beta$-stimulated fibroblasts, HNFs, HSFs and HKFs were maintained in the absence or presence of $5 \mu \mathrm{g} / \mathrm{ml}$ TGF- $\beta 1$ and $50 \mu \mathrm{mol} / 1 \mathrm{AG} 490$ 

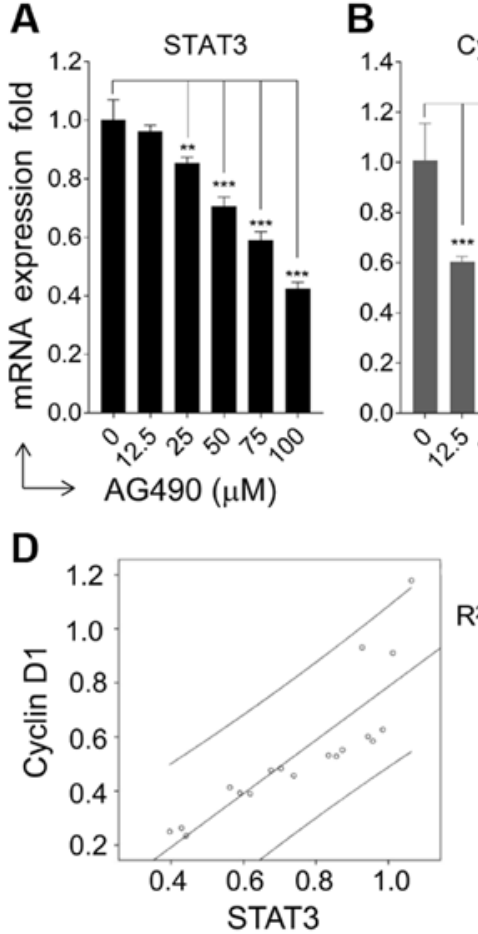

$\mathbf{F}$

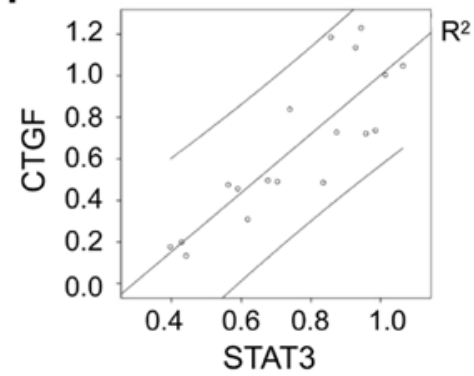

B

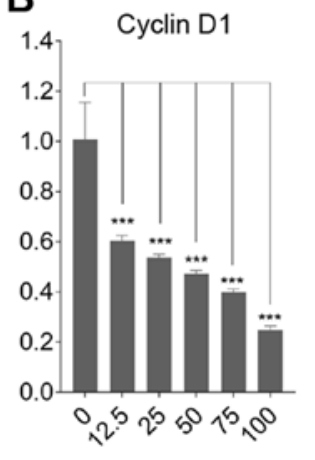

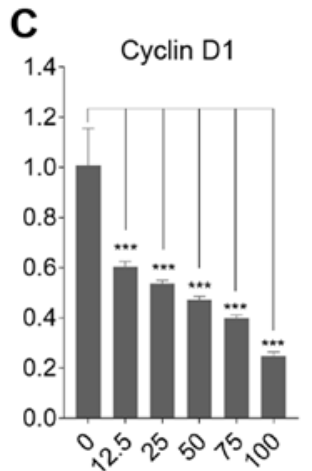

E

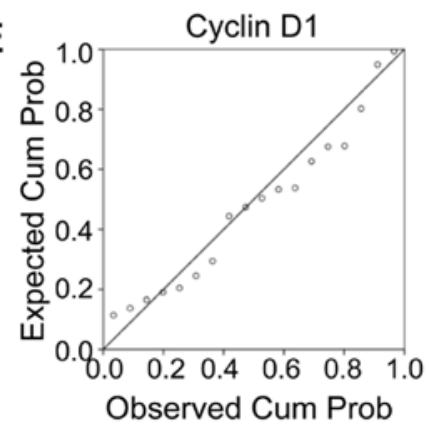

G

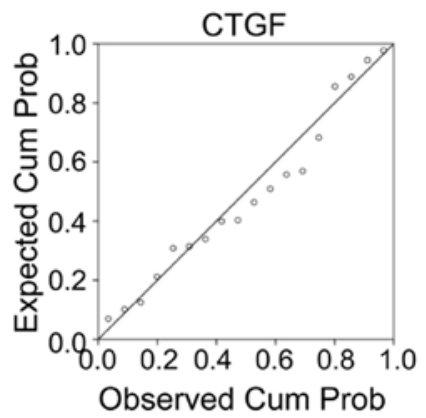

Figure 4. AG490 decreases the mRNA expression levels of STAT3, cyclin D1 and CTGF in HKFs. HKFs were treated with AG490 at concentrations of 0, 12.5 , 25, 50, 75, and $100 \mu \mathrm{mol} / \mathrm{l}$ for $24 \mathrm{~h}$. Cells were then collected and total RNA was isolated, respectively. (A-C) The mRNA expression levels for (A) STAT3, (B) cyclin D1 and (C) CTFG were quantified by RT-qPCR. Values represent the means \pm SD, $n=3$; ${ }^{* *} \mathrm{P}<0.01$ and ${ }^{* * * *} \mathrm{P}<0.005$ vs. control. (D-G) Simple linear regression analysis revealed that cyclin D1 and CTGF mRNA expression were highly associated with STAT3 mRNA levels in HKFs that were treated with AG490 at various concentrations. To efficiently deduce the linear regression model, the SPSS ${ }^{\circledR}$ Statistics software platform was used. In the regression model, STAT3 was used as a predictor (independent variable), while cyclin D1 and CTGF were used as the dependent variables. (D and F) The scatter plots represented relative mRNA expression levels of cyclin D1 (D) and CTGF (F) against that of STAT3 in AG490-treated HKF cells. The F-values in one-way ANOVA test were 43.647 for cyclin D1 and 42.138 for CTGF. The P-values were both $<0.001$. The coefficients of determination $\left(\mathrm{R}^{2}\right)$ were 0.732 for cyclin D1 and 0.725 for CTGF. The residuals were calculated and used for subsequent analysis. (E and G) The predicted probability (P-P) plots of standardized residuals were established for normality and homoscedasticity analysis. The plotted points (residuals) of (E) cyclin D1 and (G) CTGF were both evenly distributed and followed the straight lines, suggesting the regression model was valid. Observed Cum Prob, observed cumulative probability. Expected Cum Prob, expected cumulative probability. STAT3, signal transducer and activator of transcription 3; CTGF, connective tissue growth factor; HKFs, human keloid fibroblasts.

for $24 \mathrm{~h}$ before assessing cell viability. The results revealed that the TGF- $\beta 1$-induced excessive proliferation was markedly inhibited by the application of AG490 by $16.7 \%$ (HNFs), $21.2 \%$ (HSFs) and $36.2 \%$ (HKFs) ( $<<0.05$; Fig. 5A). The HKFs and HSFs exhibited higher levels of the constitutive protein expression of total STAT3 and CTGF than the HNFs (Fig. 5B and D). Following treatment with AG490, the expression levels of both STAT3 and phosphorylation (p-STAT3) were decreased in the HNFs, HSFs and HKFs that were stimulated with TGF- $\beta 1$ (Fig. 5B-D). These results were consistent with the above-mentioned findings that AG490 dose-dependently reduced both the expression and phosphorylation of STAT-3 in the HNFs and HKFs (Fig. 1). In addition, the inhibitory effects of AG490 were observed on the TGF- $\beta 1$-induced production of CTGF in fibroblasts (Fig. 5B and C). The relative mRNA expression of CTGF in HNFs, HSFs and HKFs was decreased by approximately 45,39 and $51 \%$, respectively $(\mathrm{P}<0.05$; Fig. 5E).

STAT3 ODNs inhibit HKF invasion. The delivery of the decoy ODNs into the cell has been reported as a promising strategy with which to hinder the transcription of disease related genes $(28,29)$. The ODNs usually consist of cis-element sequences that serve as the decoy for the target transcription factor and then reduce its transcriptional activity. In the present study, to assess the effects of STAT3 decoy ODNs on KFs, SODNs were transfected into HKFs in vitro. The results of EMSA demonstrated that SODNs 
A

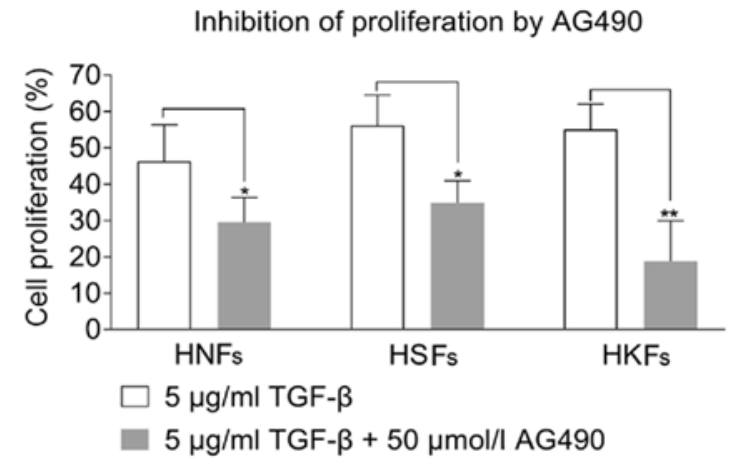

B
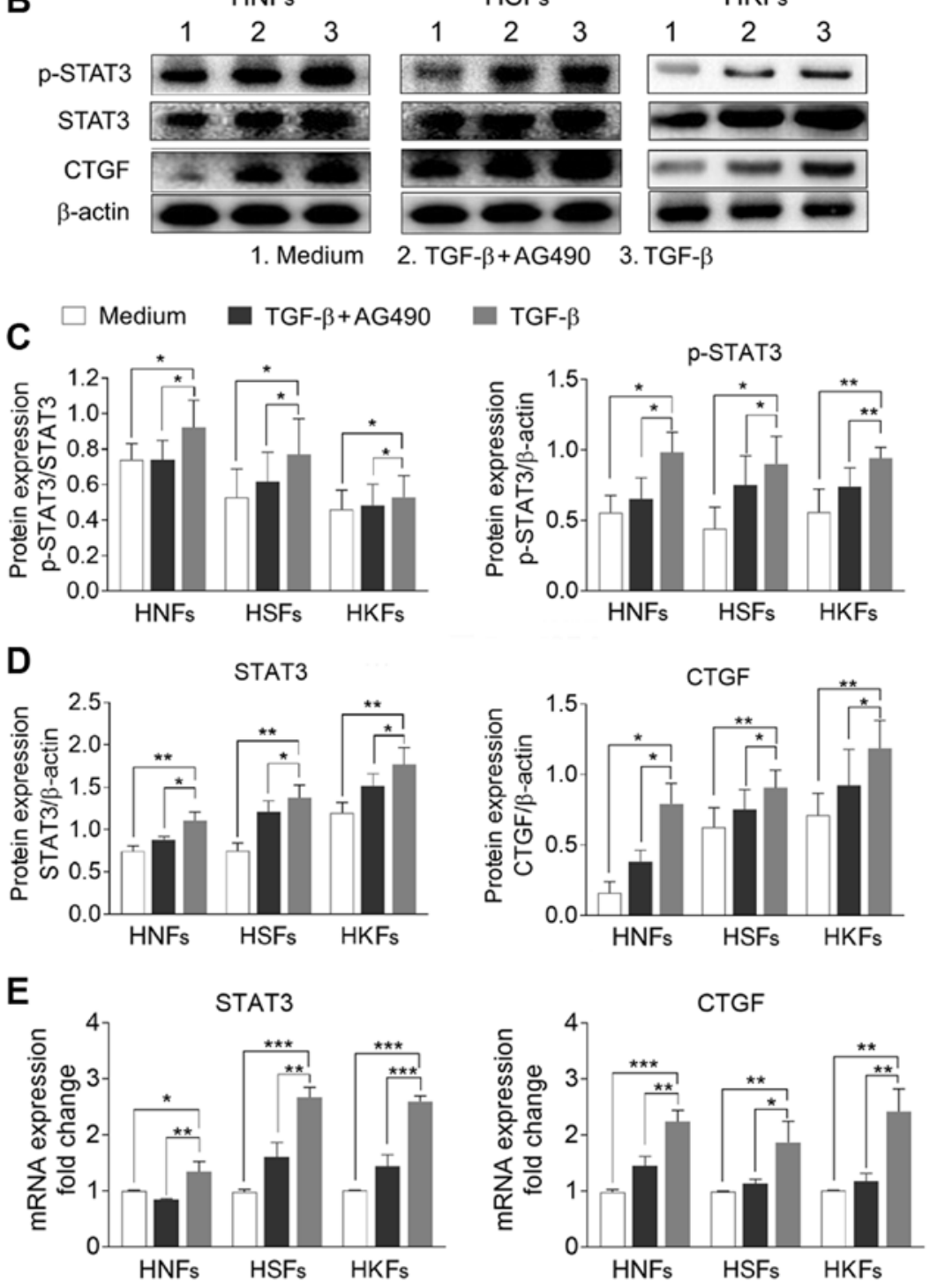

Figure 5. AG490 significantly inhibits the effects of TGF- $\beta$ on HNFs, HSFs and HKFs. HNFs, HSFs and HKFs were incubated with TGF- $\beta$ (5 $\mu \mathrm{g} / \mathrm{ml})$ for $2 \mathrm{~h}$, followed by treatment with AG490 (50 $\mu \mathrm{mol} / \mathrm{l})$ or not. HNFs. The control HSFs and HKFs were incubated with medium in the absence of either TGF- $\beta$ or AG490. (A) AG490 inhibited TGF- $\beta$-induced excessive proliferation of HNFs, HSFs and HKFs. After $24 \mathrm{~h}$ of treatment, cells were collected and the proliferation rates were detected by CCK- 8 assay. (B) AG490 reduced the expression of phosphorylated STAT3 (p-STAT3) and CTGF proteins that were promoted by TGF- $\beta$ in HNFs, HSFs and HKFs. The expression of p-STAT3 and CTGF proteins in the different groups were detected by western blot analysis. $\beta$-actin was used as a loading control. (C) The ratio of p-STAT3/STAT3 and p-STAT3/ $\beta$-actin was measured, respectively. (D) The ratio of STAT3/ $\beta$-actin and CTGF/ $\beta$-actin was measured, respectively. (E) The mRNA expression levels for total STAT3 and CTFG were quantified by RT-qPCR. Values represent the means \pm SD, $n=3$; ${ }^{*} \mathrm{P}<0.05,{ }^{* *} \mathrm{P}<0.01$ and ${ }^{* * *} \mathrm{P}<0.005$ vs. control. HNFs, human normal fibroblasts; HKFs, human keloid fibroblasts; HNFs, human normal fibroblasts.

markedly decreased the DNA-binding activity of STAT3, MONDs or control medium did not affect the activation of STAT3 (Fig. 6A). Consistent with the results obtained above, the proliferation of HKFs was also inhibited by the application of SODNs (Fig. S1C). Moreover, there was a 2 -fold decrease in the number of migrated HKFs in the presence 
A EMSA: STAT3 activity assay
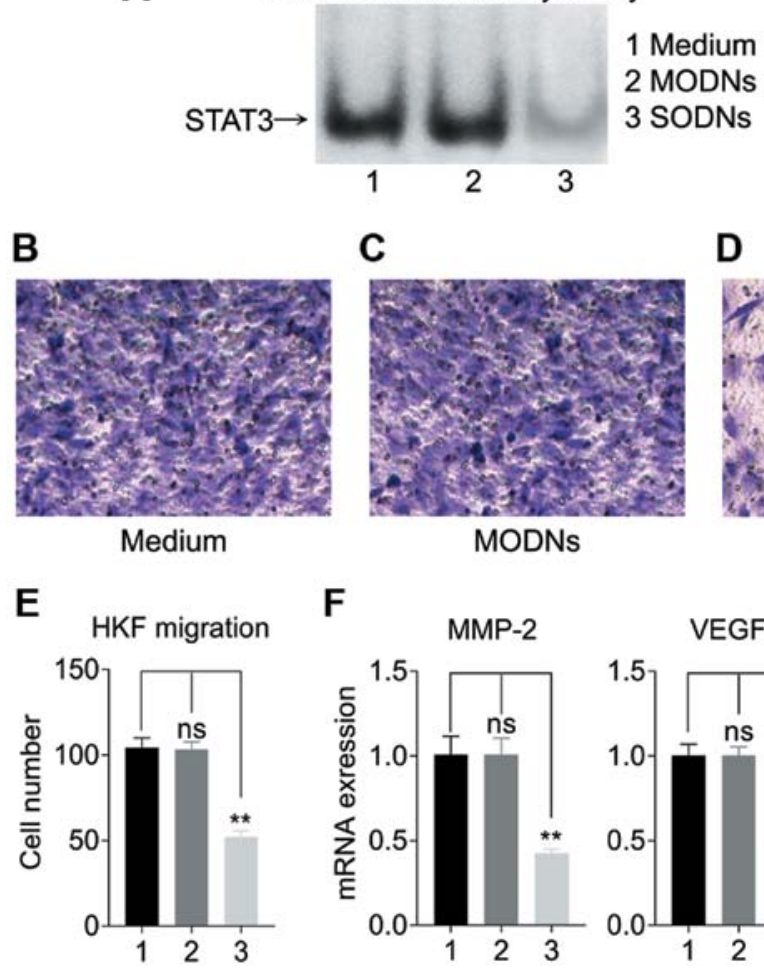

C

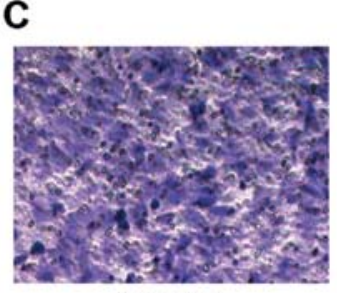

MODNs

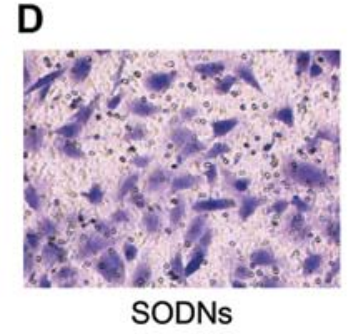

$\mathbf{F}$

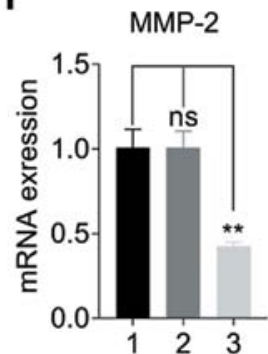

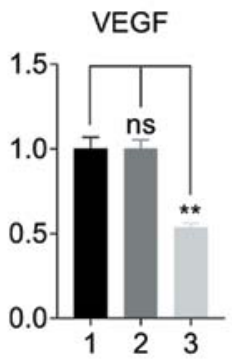

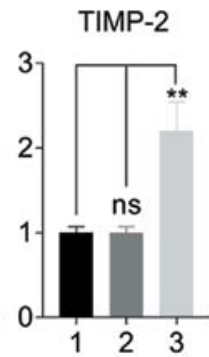

Figure 6. STAT3 decoy oligodeoxynucleotides markedly inhibit the invasion and progression of HKFs. STAT3 specific decoy oligodeoxynucleotides (SODNs) and random oligodeoxynucleotides (MODNs) were transfected into HKFs. Untransfected HKFs were used as a medium control. (A) EMSA was performed $72 \mathrm{~h}$ following transfection. SODNs blocked the activation of STAT3 signaling pathway by inhibiting the DNA-binding of activated STAT3 protein. (B-D) Cell invasion assay was performed and images are shown as representative of five independent experiments. (E) Numbers of stained cells in invasion assay were counted. Data are normalized and expressed as fold change relative to control values. (F) SODNs attenuated mRNA expression of MMP-2 and VEGF and increased mRNA expression of TIMP-2. The mRNA expression levels were quantified by RT-qPCR. Values represent the means \pm SD, $\mathrm{n}=3$; ${ }^{* *} \mathrm{P}<0.01 ; \mathrm{ns}, \mathrm{not}$ significant; STAT3, signal transducer and activator of transcription 3; HKFs, human keloid fibroblasts; MMP-2, matrix metalloproteinase 2; VEGF, vascular endothelial growth factor; TIMP-2, tissue inhibitor of metalloproteinase-2.

of SODNs (Fig. 6B-E), indicating that SODNs remarkably inhibited HKFs invasion and progression. The expression levels of ECM components, including tissue inhibitor of metalloproteinase-2 (TIMP-2), matrix metalloproteinase-2 (MMP-2) and vascular endothelial growth factor (VEGF), were detected by RT-qPCR. Transfection with SODNs also attenuated the mRNA expression of MMP-2 and VEGF by 57 and $46 \%$, respectively, and increased the expression of TIMP-2 >2-fold (Fig. 6F). These results demonstrated that decoy STAT3 can block the constitutive activation of STAT3, thereby inhibiting the invasion and progression of HKFs by upregulating the expression of TIMP-2, and downregulating that of MMP-2 and VEGF.

\section{Discussion}

The JAK/STAT signal transduction pathway plays a leading role in the regulation of diverse processes, including cell proliferation, migration, cytokine or chemokine production and inflammation (30). During the process of cutaneous wound healing, various chemokines, cytokines (such as TNF, IL-1 and IL-6) and growth factors are secreted by inflammatory cells in a well-orchestrated manner (31). The JAK/STAT signaling pathway is activated in response to most of these mediators. The enhanced expression and phosphorylation of STAT3 has been observed in keloid tissues and KFs cultured in vitro, suggesting an important role of STAT3 the pathogenesis of keloids $(12,32)$. The inhibition of STAT3 expression by siRNA or inhibitor (cucurbitacin I) has been shown to lead to corresponding decrease in collagen expression, and in the proliferation and migration of KFs (12). STAT3 can be used as a therapeutic target for keloids.

AG490, as a potent inhibitor of the JAK2/STAT3 pathway, inhibits the proliferation of HKFs effectively. It has been reported that AG490 can inhibit epidermal growth factor receptor (EGFR) in cell-free assays (33). It has previously been demonstrated that KFs exhibit a higher expression and phosphorylation levels of molecules associated with tyrosine phosphorylation signaling, including EGFR, compared with normal dermal fibroblasts (34). As a tyrosine kinase blocker, AG490 may significantly suppress KF growth by exerting combined inhibitory effects on EGFR and STAT3. The reduction of EGFR and STAT3 activation can also suppress the transcription of the downstream genes. In the present study, decreasing the expression of cyclin D1 resulted in cell cycle arrest in the G1 phase and in the promotion of cell apoptosis. The downregulation of CTGF inhibited the proliferation and differentiation of HKFs, as well as the fibrotic effects of fibroblasts. Another possible mechanism for the AG490 inhibitory effect was that the production of collagen type I and type II may be decreased through the inhibition of the activation of STAT3 in HKFs, as suggested by a previous study (12). 
However, it remains unclear as to whether collagen production is reduced and which type of collagen is affected by AG490 treatment, as collagen production was not examined in the present study.

CTGF is involved in ECM remodeling, whose overexpression is closely related to the occurrence and development of proliferative or fibrotic diseases (35). In fibroblasts, CTGF expression can be induced by both TGF- $\beta$ and downstream signals of TGF- $\beta$ (36). It has been well established that the TGF- $\beta /$ Smad signaling pathway plays a critical role in the pathogenesis of keloids by promoting collagen synthesis, ECM deposition and sustained fibrosis $(37,38)$. However, blocking the TGF- $\beta /$ Smad pathway can only prevent the initiation or progression of fibrosis; it cannot effectively reverse the fibrosis that has already occurred (39). The treatment of keloids may be more optimal by inhibiting the abnormal increase in the expression of CTGF, which has been proven to be a central mediator of tissue remodeling and fibrosis and its inhibition reverses the process of fibrosis in liver and lung tissues (40). The results of the present study demonstrated that blocking STAT3 activation decreased TGF- $\beta$-induced CTGF overexpression, suggesting that AG490 may reverse the profibrotic effects of TGF- $\beta$ by regulating CTGF in KFs. The present study demonstrated that blocking the STAT3 pathway by molecular targeted drugs or gene therapy can inhibit the abnormal proliferation and invasion of HKFs and promote apoptosis, thereby treating keloids.

In conclusion, the present study demonstrated that STAT3 can be used as a therapeutic target for keloids. By inhibiting the STAT3 pathway using AG490 or decoy ODNs, the proliferation and abnormal behavior of HKFs were significantly suppressed. The TGF- $\beta 1$-stimulated activation of STAT3 and the production of CTGF were also decreased by AG490 in fibroblasts. These results suggested that AG490 plays a protective role in keloids mainly by inducing G1 phase arrest, promoting apoptosis and suppressing inflammatory responsiveness. AG490, as a selective inhibitor of the JAK2/STAT3 pathway, may be a therapeutic candidate for inhibiting the development of keloids.

\section{Acknowledgements}

Not applicable.

\section{Funding}

The present study was supported by the Natural Science Foundation of Jiangsu Province (grant no. BK20171117).

\section{Availability of data and materials}

All data generated or analyzed during this study are included in this published article.

\section{Authors' contributions}

JB and WF designed the study. YZ and YS performed the experiments and drafted the manuscript. WH and LM participated in data analysis. YT, DL and CX were involved in the discussion and interpretation of the results. All authors read and approved the final manuscript.

\section{Ethics approval and consent to participate}

Not applicable.

\section{Patient consent for publication}

Not applicable.

\section{Competing interests}

The authors declare that they have no competing interests.

\section{References}

1. Shih B, Garside E, McGrouther DA and Bayat A: Molecular dissection of abnormal wound healing processes resulting in keloid disease. Wound Repair Regen 18: 139-153, 2010.

2. Cosman B, Crikelair GF, Ju DMC, Gaulin JC and Lattes R: The surgical treatment of keloids. Plast Reconstr Surg 27: 335-358, 1961.

3. Viera MH, Vivas AC and Berman B: Treatment of keloids and scars. Ethn Dermatology Princ Pract: 159-172, 2013.

4. Tuan TL and Nichter LS: The molecular basis of keloid and hypertrophic scar formation. Mol Med Today 4: 19-24, 1998.

5. Ledon JA, Savas J, Franca K, Chacon A and Nouri K: Intralesional treatment for keloids and hypertrophic scars: A review. Dermatol Surg 39: 1745-1757, 2013.

6. Manca G, Pandolfi P, Gregorelli C, Cadossi M and de Terlizzi F: Treatment of keloids and hypertrophic scars with bleomycin and electroporation. Plast Reconstr Surg 132: 621e-630e, 2013.

7. Vincent AS, Phan TT, Mukhopadhyay A, Lim HY, Halliwell B and Wong KP: Human skin keloid fibroblasts display bioenergetics of cancer cells. J Invest Dermatol 128: 702-709, 2008.

8. Naitoh M, Hosokawa N, Kubota H, Tanaka T, Shirane H, Sawada M, Nishimura Y and Nagata K: Upregulation of HSP47 and collagen type III in the dermal fibrotic disease, keloid. Biochem Biophys Res Commun 280: 1316-1322, 2001

9. Uitto J, Perejda AJ, Abergel RP, Chu ML and Ramirez F: Altered steady-state ratio of type I/III procollagen mRNAs correlates with selectively increased type I procollagen biosynthesis in cultured keloid fibroblasts. Proc Natl Acad Sci USA 82: 5935-5939, 1985.

10. Babu M, Diegelmann R and Oliver N: Keloid fibroblasts exhibit an altered response to TGF-beta. J Invest Dermatol 99: 650-655, 1992.

11. Banerjee $\mathrm{K}$ and Resat $\mathrm{H}$ : Constitutive activation of STAT3 in breast cancer cells: A review. Int J Cancer 138: 2570-2578, 2016.

12. Lim CP, Phan TT, Lim IJ and Cao X: Stat 3 contributes to keloid pathogenesis via promoting collagen production, cell proliferation and migration. Oncogene 25: 5416-5425, 2006.

13. Abroun S, Saki N, Ahmadvand M, Asghari F, Salari F and Rahim F: STATs: An old story, yet mesmerizing. Cell J 17: 395-411, 2015.

14. Schlessinger K and Levy DE: Malignant transformation but not normal cell growth depends on signal transducer and activator of transcription 3. Cancer Res 65: 5828-5834, 2005.

15. Meydan N, Grunberger T, Dadi H, Shahar M, Arpaia E, Lapidot Z, Leeder JS, Freedman M, Cohen A, Gazit A, et al: Inhibition of acute lymphoblastic leukaemia by a Jak-2 inhibitor. Nature 379: 645-648, 1996.

16. An JY, Pang HG, Huang TQ, Song JN, Li DD, Zhao YL and Ma XD: AG490 ameliorates early brain injury via inhibition of JAK2/STAT3-mediated regulation of HMGB1 in subarachnoid hemorrhage. Exp Ther Med 15: 1330-1338, 2018.

17. Xu MY, Hu JJ, Shen J, Wang ML, Zhang QQ, Qu Y and Lu LG: Stat3 signaling activation crosslinking of TGF- $\beta 1$ in hepatic stellate cell exacerbates liver injury and fibrosis. Biochim Biophys Acta 1842: 2237-2245, 2014.

18. Nielsen M, Kaltoft K, Nordahl M, Röpke C, Geisler C, Mustelin T, Dobson P, Svejgaard A and Odum N: Constitutive activation of a slowly migrating isoform of Stat 3 in mycosis fungoides: Tyrphostin AG490 inhibits Stat3 activation and growth of mycosis fungoides tumor cell lines. Proc Natl Acad Sci USA 94: 6764-6769, 1997. 
19. Burdelya L, Catlett-Falcone R, Levitzki A, Cheng F, Mora LB, Sotomayor E, Coppola D, Sun J, Sebti S, Dalton WS, et al: Combination therapy with AG-490 and interleukin 12 achieves greater antitumor effects than either agent alone. Mol Cancer Ther 1: 893-899, 2002.

20. Samanta AK, Lin H, Sun T, Kantarjian H and Arlinghaus RB: Janus kinase 2: A critical target in chronic myelogenous leukemia. Cancer Res 66: 6468-6472, 2006.

21. Abe M, Funakoshi-Tago M, Tago K, Kamishimoto J, Aizu-Yokota E, Sonoda Y and Kasahara T: The polycythemia vera-associated Jak2 V617F mutant induces tumorigenesis in nude mice. Int Immunopharmacol 9: 870-877, 2009.

22. Ma J, Yan X, Lin Y and Tan Q: Hepatocyte growth factor secreted from human adipose-derived stem cells inhibits fibrosis in hypertrophic scar fibroblasts. Curr Mol Med, Jan 5, 2020 (Epub ahead of print).

23. Wu X, Bian D, Dou Y, Gong Z, Tan Q, Xia Y and Dai Y: Asiaticoside hinders the invasive growth of keloid fibroblasts through inhibition of the GDF-9/MAPK/Smad pathway. J Biochem Mol Toxicol 31, 2017.

24. Livak KJ and Schmittgen TD: Analysis of relative gene expression data using real-time quantitative PCR and the 2(-Delta Delta C(T)) method. Methods 25: 402-408, 2001

25. Dunkel Y, Ong A, Notani D, Mittal Y, Lam M, Mi X and Ghosh P STAT3 protein up-regulates $\mathrm{G} \alpha$-interacting vesicle-associated protein (GIV)/Girdin expression, and GIV enhances STAT3 activation in a positive feedback loop during wound healing and tumor invasion/metastasis. J Biol Chem 287: 41667-41683, 2012.

26. Wlodkowic D, Telford W, Skommer J and Darzynkiewicz Z: Apoptosis and beyond: Cytometry in studies of programmed cell death. Methods Cell Biol 103: 55-98, 2011.

27. Pakyari M, Farrokhi A, Maharlooei MK and Ghahary A: Critical role of transforming growth factor beta in different phases of wound healing. Adv Wound Care (New Rochelle) 2: 215-224, 2013

28. Hanagata $\mathrm{N}$ : $\mathrm{CpG}$ oligodeoxynucleotide nanomedicines for the prophylaxis or treatment of cancers, infectious diseases, and allergies. Int J Nanomedicine 12: 515-531, 2017.

29. Tomita N, Ogihara T and Morishita R: Transcription factors as molecular targets: Molecular mechanisms of decoy ODN and their design. Curr Drug Targets 4: 603-608, 2003.

30. Rawlings JS, Rosler KM and Harrison DA: The JAK/STAT signaling pathway. J Cell Sci 117: 1281-1283, 2004.

31. Efron PA and Moldawer LL: Cytokines and wound healing: The role of cytokine and anticytokine therapy in the repair response. J Burn Care Rehabil 25: 149-160, 2004
32. Ghazizadeh M, Tosa M, Shimizu H, Hyakusoku $H$ and Kawanami O: Functional implications of the IL-6 signaling pathway in keloid pathogenesis. J Invest Dermatol 127: 98-105, 2007.

33. Gazit A, Osherov N, Posner I, Yaish P, Poradosu E, Gilon C and Levitzki A: Tyrphostins. 2. Heterocyclic and alpha-substituted benzylidenemalononitrile tyrphostins as potent inhibitors of EGF receptor and ErbB2/neu tyrosine kinases. J Med Chem 34: 1896-1907, 1991.

34. Chin GS, Liu W, Steinbrech D, Hsu M, Levinson H and Longaker MT: Cellular signaling by tyrosine phosphorylation in keloid and normal human dermal fibroblasts. Plast Reconstr Surg 106: 1532-1540, 2000

35. Lipson KE, Wong C, Teng Y and Spong S: CTGF is a central mediator of tissue remodeling and fibrosis and its inhibition can reverse the process of fibrosis. Fibrogenesis Tissue Repair 5 (Suppl 1): S24, 2012.

36. Jurzak M, Adamczyk K, Antończak P, Garncarczyk A, Kuśmierz D and Latocha M: Evaluation of genistein ability to modulate CTGF mRNA/protein expression, genes expression of TGF $\beta$ isoforms and expression of selected genes regulating cell cycle in keloid fibroblasts in vitro. Acta Pol Pharm 71: 972-986, 2014.

37. Biernacka A, Dobaczewski $M$ and Frangogiannis NG: TGF- $\beta$ signaling in fibrosis. Growth Factors 29: 196-202, 2011.

38. Walton KL, Johnson KE and Harrison CA: Targeting TGF- $\beta$ mediated SMAD signaling for the prevention of fibrosis. Front Pharmacol 8: 461, 2017.

39. Song R, Li G and Li S: Aspidin PB, a novel natural anti-fibrotic compound, inhibited fibrogenesis in TGF- $\beta 1$-stimulated keloid fibroblasts via PI-3K/Akt and Smad signaling pathways. Chem Biol Interact 238: 66-73, 2015.

40. Bickelhaupt S, Erbel C, Timke C, Wirkner U, Dadrich M, Flechsig P, Tietz A, Pföhler J, Gross W, Peschke P, et al: Effects of CTGF blockade on attenuation and reversal of radiation-induced pulmonary fibrosis. J Natl Cancer Inst 109, 2017.

(i) $\Theta$ This work is licensed under a Creative Commons Attribution-NonCommercial-NoDerivatives 4.0 International (CC BY-NC-ND 4.0) License. 